\title{
Star Cluster Migration Near the Galactic Center
}

\section{Michiko Fujii $^{12}$, Masaki Iwasawa ${ }^{2}$, Yoko Funato $^{3}$, and Junichiro Makino ${ }^{2}$}

${ }^{1}$ Depertment of Astronomy, Graduate School of Science, The University of Tokyo, Japan

${ }^{2}$ Division of Theoretical Astronomy, National Astronomical Observatory of Japan, Japan

${ }^{3}$ Department of General System Studies, College of Arts and Sciences, The University of Tokyo, Japan

\begin{abstract}
We performed a self-consistent $N$-body simulation of star clusters in the Galactic center (GC), taking into account the collisions of stars and formation of an intermediate-mass black hole (IMBH). We find that if an IMBH forms in the cluster, it carries young stars to the GC by a 1:1 resonance.
\end{abstract}

Keywords. Galaxy: center, Galaxy: kinematics and dynamics, galaxies: star clusters

There are two feeding processes for the growth of supermassive black holes (BHs). One is direct gas accretion and the other is in-spiraling of stars. In the central region of our Galaxy, young stars and groups of stars, IRS13E, which look like a remnant of a star cluster, have been observed. For these stars, two formation scenarios have been suggested; in-situ formation in the accretion disk (Levin \& Beloborodov 2003) and star cluster migration (Gerhard 2001). Some simulations for the in-situ formation have been performed. However, it is difficult to form S-stars within a few thousand AU of the GC. On the other hand, in our Galaxy, two young clusters are found at around $30 \mathrm{pc}$ from the Galactic center. We performed fully self-consistent $N$-body simulations of a star cluster and its parent galaxy using a tree-direct hybrid code, BRIDGE (Fujii et al. 2007). In our simulation, an $\mathrm{IMBH}$ is formed in the star cluster and it migrates into the GC. This IMBH traps many stars in its 1:1 mean motion resonance and carries them to the GC (Fujii et al. 2009). The stars carried to the center through the 1:1 resonance are typically much more massive than average, since stars which have sunk to the center of the cluster are selectively trapped. In AGNs with nuclear starbursts, stars may be fed to the central BH via this mechanism.

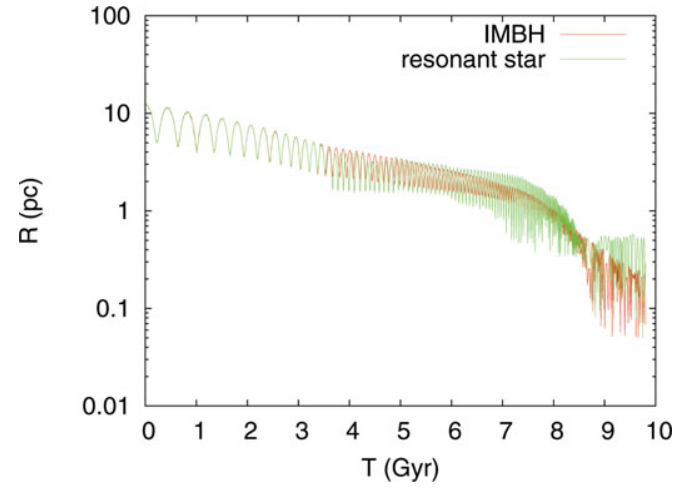

\section{References}

Fujii, M., Iwasawa, M., Funato, Y., \& Makino, J. 2007, PASJ, 59, 1095

Fujii, M., Iwasawa, M., Funato, Y., \& Makino, J. 2009, ApJ, 695, 1421

Gerhard, O. 2001, ApJ, 546, L39

Levin, Y. \& Beloborodov, M. 2003, ApJ, 590, L33

Figure 1. Orbital evolution of the IMBH and a resonant star. 